\title{
\& $\begin{array}{ll}\text { Research Square } & \text { They should not be considered conclusive, used to inform clinical practice, } \\ \text { or referenced by the media as validated information. }\end{array}$ \\ Functional polymorphisms in the MED13L and SLC4A7 genes are associated with pregnancy-induced hypertension
}

\section{Ying Zhou}

Central South University Xiangya School of Medicine

\section{Xun Li}

Hunan Children's Hospital

\section{Shimin Hu}

Central South University Xiangya School of Public Health

\section{Shujin Zhou}

Central South University Xiangya School of Medicine

Hongzhuan Tan ( $\nabla$ tanhz99@qq.com )

Central South University Xiangya School of Public Health https://orcid.org/0000-0001-9764-1813

\section{Research article}

Keywords: gestational hypertension; preeclampsia; single nucleotide polymorphism; mediator complex subunit 13like; solute carrier family 4 member 7; cytochrome P450 family 21 subfamily A member 2

Posted Date: September 27th, 2019

DOl: https://doi.org/10.21203/rs.2.15312/v1

License: @ (1) This work is licensed under a Creative Commons Attribution 4.0 International License. Read Full License 


\section{Abstract}

Background: Pregnancy-induced hypertension (PIH), including gestational hypertension (GH) and preeclampsia (PE), might share pathophysiologic mechanisms and genetic risk factors with hypertension. This study aimed to investigate whether genetic risk factors for hypertension also been associated with PIH. Methods: This is a case control candidate gene association study. A total of 84 cases with $\mathrm{GH}, 108$ cases with $\mathrm{PE}$, and 192 age-matched $( \pm 1$ year) controls were recruited from the Liuyang Municipal Hospital of Maternal and Child Health, Hunan Province of China. Three candidate single nucleotide polymorphisms (SNPs) which have been found to be associated with hypertension or blood pressure (BP) were chosen. The associations between SNPs and GH and PE were analyzed by multiple logistic regression models after adjusting for fetal sex and primipara. Results: GG genotype at mediator complex subunit 13-like (MED13L) rs11067763 was a protective factor for developing GH (GG vs. AA + AG, OR = $0.376,95 \% \mathrm{Cl}=0.158-0.892)$ and $\mathrm{PE}(\mathrm{GG}$ vs. $\mathrm{AA}+\mathrm{AG}, \mathrm{OR}=0.489,95 \% \mathrm{Cl}=0.245-0.979)$ in recessive model. $A A$ genotype at solute carrier family 4 member 7 (SLC4A7) rs820430 was a risk factor for developing GH (AA vs. GG + $A G, O R=3.562,95 \% C l=1.343-9.447)$ and $P E(A A$ vs. $G G+A G, O R=3.351,95 \% C l=1.352-8.303)$ in recessive model. But no significant association was observed between cytochrome P450 family 21 subfamily A member 2 (CYP21A2) rs2021783 and the GH/PE groups. Conclusions: PIH and hypertension are likely to share genetic risk factors. The associations both between variants of MED13L rs11067763 and GH/PE and between variants of SLC4A7 rs820430 and GH/PE were significant.

\section{Background}

Pregnancy-induced hypertension ( $\mathrm{PIH}$, including gestational hypertension $[\mathrm{GH}]$ and preeclampsia [PE]) is a pregnancy-specific complication affecting 5-10\% of all pregnant women in the world [1]. Both GH and PE are characterized by hypertension first appearing after 20 weeks of gestation, while PE is also characterized by new onset proteinuria [2]. PIH is a leading cause of maternal and fetal morbidity and mortality. Without knowing the exact etiology of PIH, delivery of the baby is the only effective way when the lives of the pregnant women are threatened [3, 4].

Since familial association was observed for $\mathrm{PIH}$, inheritance could have played a major function in the pathology of $\mathrm{PIH}$. A twin study suggested the heritability of GH and PE was $24 \%$ and $54 \%$, respectively [5]. Besides, it has been reported that maternal genetic factors contribute to PIH susceptibility[6]. Epidemiological studies also have shown that a family history of chronic hypertension is more common in PE groups compared with controls, and PIH is associated with an increased long-term risk of maternal hypertension[7]. Studies on pathophysiologic mechanisms have demonstrated endothelial dysfunction, inflammation, and vascular remodeling to be crucial to the development of chronic hypertension, as well as PIH [8, 9]. In line with these findings, it has been suggested that PIH and hypertension might share pathophysiological factors. And the genetic risks predisposing to hypertension and $\mathrm{PIH}$ may thus be partially overlapping $[8,10,11]$.

Genome-wide association studies (GWAS) have been conducted to search for hypertension-susceptibility genes. Recently, Chinese researchers identified that mediator complex subunit 13-like (MED13L) rs11067763, solute carrier family 4 member 7 (SLC4A7) rs820430, and cytochrome P450 family 21 subfamily A member 2 (CYP21A2) rs2021783 were possibly genetic variants associated with blood pressure (BP) and/or hypertension[12]. Further studies found that hypertension risk SNP rs820430 functioned as an enhancer of SLC4A7 [13]. Another GWAS carried out in the USA found associations between CYP17A1/CYP1A2 and BP [14]. Considering the overlapping of genetic risks of hypertension and $\mathrm{PIH}$, it is valuable to investigate the relationship between these three SNPS and PIH. 
The present study aims to detect the association between three SNPs, which were previously reported having association with BP or hypertension, and PIH in a Southern Han Chinese population.

\section{Materials And Methods}

\section{Study population}

Statistical analyses were conducted using IBM SPSS 19.0 software (IBM SPSS, Inc., Chicago, IL). A total of 108 patients with $\mathrm{PE}, 84$ with $\mathrm{GH}$, and 192 controls were recruited from the Liuyang Municipal Hospital of Maternal and Child Health, Hunan Province of China. The inclusion criteria for the case group were clinical diagnosis of GH or PE without diabetes mellitus, renal disease, cerebrovascular disease (CVDs), or other diseases that are already known as risk factors for $\mathrm{PIH}$, and HELLP syndrome was excluded. The inclusion criteria for the control group were agematched ( \pm 1 year) normotensive pregnant women who delivered at the same hospital during the same period and with the absence of aforementioned diseases.

\section{Ethics statement}

This study conducted in accordance with the 1975 Declaration of Helsinki and was reviewed and approved by the Committee for Human Research Protections of Xiang-Ya School of Medicine. Informed consent was obtained from each individual included in the study, after which blood samples and medical records were collected.

\section{Diagnostic criteria}

PE was defined according to the International Society for Study of Hypertension in Pregnancy as having de novo hypertension (systolic blood pressure[SBP] $\geq 140 \mathrm{mmHg}$ or diastolic blood pressure[DBP] $\geq 90 \mathrm{mmHg}$ after the 20th week of gestation) and accompanying proteinuria (urinary protein dip sticks $\geq 2+$ or $\geq 300 \mathrm{mg}$ in a 24-h urine sample). GH was similarly defined as de novo hypertension without proteinuria $[15,16]$.

\section{SNP selection and genotyping}

Three candidate SNPs were selected, including MED13L rs11067763, SLC4A7rs820430, and CYP21A2 rs2021783. Genomic DNA was extracted from whole blood using the TIANamp Blood DNA Kit (DP318-03, TIANGEN, Beijing). Genotyping of the three SNPs was performed using matrix-assisted laser desorption/ionization-time of flight(MALDITOF) mass spectrometry, according to the manufacturer's instructions[17].

\section{Statistical analysis}

Statistical analyses were conducted using IBM SPSS 19.0 software (IBM SPSS, Inc., Chicago, IL). Mean \pm standard deviation (SD), or median and interquartile were used to describe the continuous variables with normal or skewed distribution, respectively. Frequency was used to describe categorical variables. To compare demographic and clinical data, the t-test or Wilcoxon rank sum test was used for continuous variables, and the Chi-square test or Fisher's exact test was used for categorical variables. The Bonferroni correction was applied for multiple comparisons $(a=0.05 / 3=0.0167)$. The SNP detection rate was calculated as the number of sites that were successfully genotyped for all of the samples divided by the number of genotyped sites for all samples. The HardyWeinberg test was conducted in control groups using the Chi-square goodness-of-fit test or the Fisher's exact test (a $=0.01$ ). The Chi-square test or Fisher's exact test was used to test the allele/genotype distribution between the case 
and control groups. Univariate logistic regression was used to estimate the crude odds ratio (OR) and its corresponding $95 \%$ confidence interval $(95 \% \mathrm{Cl})$ for different genetic models, including dominant model and recessive model. Through multiple logistic regression, the adjusted $\mathrm{OR}$ and $95 \% \mathrm{Cl}$ were also calculated after adjusting for fetal sex and pimipara[18, 19].

\section{Results}

\section{Demographic and clinical features}

In total, $84 \mathrm{GH}$ cases, 108 PE cases, along with 192 controls were analyzed. The main demographic and clinical features of three groups are summarized in Table 1. No significant between-group differences were detected for fetal sex, primipara, and new born weight $(P>0.0167)$. Meanwhile, serum uric acid, in-hospital SBP, and in-hospital DBP with $\mathrm{GH}$ and PE were significantly higher than those in normotensive women. Moreover, in-hospital SBP of PE women were significantly higher than those in $\mathrm{GH}$ women, whereas there were no significant differences between the $\mathrm{GH}$ and PE groups as to other characteristics. Interestingly, creatinine of women with PE were significantly higher than those in $\mathrm{GH}$ and control groups, and gestational age of PE groups were significantly lower than those in $\mathrm{GH}$ and control groups, while no significant differences were found between $\mathrm{GH}$ and control groups on these two factors.

\section{SNPs and PIH}

The SNP detection rate was $98 \%$. The allele frequencies of control groups showed no deviation from Hardy-Weinberg equilibrium, shown the representativeness of our control group. The distributions of genotypes and alleles among the three groups are shown in Table 2. The results of the Chi-square test showed that the distribution of MED13L rs11067763 genotype was significantly different between the $\mathrm{GH}$ and control groups $(P=0.007)$, and there was significant difference in the SLC4A7 rs820430 allele between the $\mathrm{GH}$ and control groups $(P=0.043)$, but no significant differences in these two SNPs genotype/allele were observed between the PE and control groups. Furthermore, no significant differences in CYP21A2 rs2021783 genotype/allele were found between the case and control groups.

The univariate logistic regression results are shown in Table 3. Two SNPs showed nominal association with GH or PE. Specifically, MED13L rs11067763 was significantly associated with both GH (GG vs. AA + AG, $P=0.009)$ and PE (GG vs. AA + AG, $P=0.034$ ) in recessive model. Also, SLC4A7rs820430 was significantly associated with both $\mathrm{GH}$ (AA vs. GG, $P=0.021$; AA vs. GG + AG, $P=0.020$ ) and $P E(A A$ vs. $G G+A G, P=0.026)$. However, no significant association was observed between CYP21A2 rs2021783 and the GH/PE groups.

After adjusted for fetal sex and primipara by logistic regression, most of the results remained the same as the univariate logistic regression results (Table 3). MED13L rs11067763 was significantly associated with both GH and $P E$, being a carrier of the $G G$ genotype was associated with a decreased susceptibility of developing GH/PE (GG vs. $\mathrm{AA}+\mathrm{AG}, P=0.027$; and GG vs. AA + AG, $P=0.043$, respectively ); $S L C 4 A 7$ rs820430 was significantly associated with both $\mathrm{GH}$ and $\mathrm{PE}$, being a carrier of the $A A$ genotype was associated with an increased susceptibility of developing GH/PE (AA vs. GG, $P=0.007$, AA vs. GG + AG, $P=0.011$; and AA vs. GG, $P=0.026, \mathrm{AA}$ vs. GG $+\mathrm{AG}, P=$ 0.009 , respectively); being a carrier of the SLC4A7 rs820430 A allele was associated with an increased susceptibility of developing GH (A vs. G, $P=0.033$ ). However, still no significant association was detected between CYP21A2 rs2021783 and the GH/PE groups in multiple logistic regression analysis.

\section{Discussion}


The typical manifestation of PIH is BP elevation. Women with a history of PIH are more susceptible to chronic hypertension囚and women with chronic hypertension are at increased risk for PE. Luscher demonstrated that endothelial cell injury in PIH women could profoundly affect cardiovascular function, and patients were likely to become hypertensive, hyperlipidemic, and eventually develop atherosclerosis with aging[7]. Ellen et al. reported that women with chronic hypertension had an increased rate of PE (17-25\%, vs. 3-5\% in the general population)[20]. Women with chronic arterial hypertension were also at increased risk for superimposed PE [21]. Except for similar metabolic bases, $\mathrm{PIH}$ and hypertension share some genetic risk factors. Our team had found significant associations between angiotensinogen ( $A G T)$ rs3789678 and $\mathrm{GH}$ and between angiotensin II receptor type 1 (AGTR1) rs275645 and PE after adjusting for fetal sex, maternal age, and gestational diabetes mellitus (GDM) [11]. So validate the association between hypertension candidate genes and $\mathrm{PIH}$ is a reasonable and effective way to find genetic risk factors for PIH.

In this case-control study, significant differences were found between MED13L rs11067763 and GH/PE, and SLC4A7 rs820430 associated with the onset and development of GH/PE. In detail, carrying the GG genotype at rs11067763 was a protective factor for developing GH/PE. As for rs820430, carrying the AA genotype was a risk factor for developing GH/PE. But no significant association between CYP21A2 rs2021783 polymorphisms and GH/PE was found. Importantly, our study reveals for the first time that the MED13L rs11067763 and SLC4A7rs820430 were associated with GH/PE risk among Chinese Han women.

MED13L is a paralog of the closely related protein named Mediator complex subunit 13 (MED13), which is believed to have arisen from gene duplication events[22]. It is responsible for bridging the RNA pol II enzyme and transcription factors to initiate transcription of protein-coding genes and non-coding RNA genes [23]. Through retinoblastoma $(\mathrm{Rb}) / \mathrm{E} 2 \mathrm{~F}$ pathways, it promotes proliferation of vascular smooth muscle cells which are important in the development of hypertension [24]. Besides, several researches have shown an indirect association between MED13L and inflammation through Wnt pathway which plays a regulatory role in immune system[25]. Moreover, MED13L regulates the expression level of activating transcription factor 4 (ATF4) which regulates the secretion of proinflammatory cytokines such as interleukin (IL)-6 and IL-8[26, 27], and these two cytokines have been considered influencing the onset of GH and PE. As Adela et al. reported, the serum level of IL-6 decreased in GH subjects [28]. Besides, Krasnyi found that increased endothelial IL-8 expression represented endothelial dysfunctions, which are significant pathophysiologic features of PE [29]. In our study, we found genetic variants of MED13L rs11067763 associated with development of GH/PE in Chinese Han women. GG genotype at rs 11067763 has shown to be a protective factor for GH/PE. Thus, more studies are needed to validate the association in larger population, and researches on mechanisms are necessary to uncover the molecular pathways of the association.

SLC4A7 gene is on chromosome 3p24.1, it belongs to the SLC4 family [30]. SLC4A7 encodes the electroneutral $\mathrm{Na}^{+} / \mathrm{HCO}_{3}^{-}$, co-transporter NBCn1. Several researchers have demonstrated that $\mathrm{NBCn} 1$ has a role in controlling intracellular $\mathrm{pH}(\mathrm{pHi})$ in vascular smooth muscle cells (VSMCs), and pHi is a determinant of VSMCs contractility and endothelial function, both of which effect BP. Furthermore, through elevated steady-state pHi and accelerated recovery from intracellular acidosis, they also found genetic variants of SLC4A7 rs13082711 associated with increased SLC4A7 expression level and NBCn1 function.[31-34]. A large scale GWAS also detected association between polymorphisms of SLC4A7rs13082711 and SBP/DBP [35]. A study included 275 participants from rural district of Shandong Province of China indicated rs820430 functioned as an enhancer of SLC4A7 [13]. Besides, NBCn1 promotes arterial remodeling which can cause luminal narrowing and obstruct blood flow [36]. It is known to us that increased vascular resistance[37], invasion of placental trophoblast, and failed remodeling of uterine spiral arterial[38] are pathological bases of $\mathrm{PIH}$. Therefore variants of $S L C 4 A 7$ might associate with the onset of $\mathrm{PIH}$ 
through several pathways such as BP regulation, and remodeling of arterial. We reported the polymorphisms of SLC4A7 rs820430 was associated with the onset of GH and PE in Chinese Han women for the first time. AA genotype at rs820430 is a risk genotype for $\mathrm{GH}$ and $\mathrm{PE}$, and A allele at rs 820430 is a risk allele for $\mathrm{GH}$. However, more studies are needed to identify pathophysiological path from $\mathrm{PIH}$ associated genetic variation, to gene expression, and subsequently to gene function of $S L C 4 A 7$ that alters cellular behavior.

CYP21A2 is located on chromosome 6 within the HLA (human leukocyte antigen) locus. It encodes 21-hydroxylase enzyme, a member of the cytochrome P450 (CYP450) superfamily [39]. 21-hydroxylase enzyme is necessary to the synthesis of cortisol and aldosterone, the latter regulates the amount of salt retained by the kidneys and then affects blood volume of body and BP. The mutation of CYP21A2 is related to congenital adrenal hyperplasia (CAH) [40]. Many studies have investigated the consequences of the mutations of the CYP450 superfamily. Though Chatuphonprasert et al. found the association between PE and altered expression of CYP27A1 protein in placenta, maternal, and/or fetal serum [41], another research conducted in a Korean population detected no significant association between polymorphisms in CYP1A7gene and PE [42]. Also, a Spanish study included 500 Caucasians from the region of Asturias indicated that the variation in CYP3A5, CYP3A4, and CYP21A2 did not contribute to the risk for PIH [43]. Similarly, in our study no significant association has been observed between variants of CYP21A2 rs2021783 and PIH.

Our case-control study was limited by the small sample size. It is difficult to identify very weak associations between SNP and PIH. This issue is common in gene-association studies. Although we did not detect significant difference between fetal sex and PIH, the proportions of female fetus in GH and PE groups were higher than those in control group. Different from the systematic review[44], we found primipara were less likely to develop PE. It was due to the unequal proportion of primipara and multipara in our study. In order to detect accurate relations, we still set fetal sex and primipara as covariates in multiple logistic regression. Another limitation of our study is that the gestational age of PE onset and medical regimens were not regularly recorded in the medical records of our study region, we did not conduct subgroup analyses according to early- or late-onset PE and did not categorize PE cases by severity. Also, we did not adjust for family history of hypertension or PIH and pre-pregnancy body mass index (BMI) in the multiple logistic regression because the proportion of missing data for it was larger than $15 \%$. Further researches with larger sample size and more comprehensive clinical characteristics are necessary to investigate the disease-related genes in $\mathrm{GH}$ and different types of PE.

\section{Conclusions}

In conclusion, $\mathrm{PIH}$ and hypertension may share genetic factors, which provide potential genetic evidence for patients with PIH having a higher risk for hypertension. Our study reported for the first time that the associations between variants of MED13L rs11067763 and GH and between variants of SLC4A7 rs820430 and GH/PE were significant. Further studies are needed to uncover the molecular mechanisms of these associations.

\section{Abbreviations}

Pregnancy-induced hypertension, (PIH); gestational hypertension, (GH); preeclampsia, (PE); single nucleotide polymorphism, (SNP); Genome-wide association studies, (GWAS); mediator complex subunit 13-like (MED13L); solute carrier family 4 member 7, (SLC4A7); cytochrome P450 family 21 subfamily A member 2, (CYP21A2); systolic blood pressure, (SBP); diastolic blood pressure, (DBP); cerebrovascular disease, (CVDs); body mass index, (BMI); angiotensinogen, (AGT); angiotensin II receptor type 1 (AGTR1); gestational diabetes mellitus, (GDM); activating 
transcription factor 4, (ATF4); vascular smooth muscle cells, (VSMCs); human leukocyte antigen, (HLA); congenital adrenal hyperplasia, $(\mathrm{CAH})$

\section{Declarations}

\section{Ethics approval and consent to participate}

The study was reviewed and approved by the Committee for Human Research Protections of Xiang-Ya School of Medicine. Informed consent was obtained from each individual included in the study, after which blood samples and medical records were collected.

\section{Consent for publication}

Not appllicable.

\section{Availability of data and materials}

The datasets used and/or analysed during the current study are available from the corresponding author on reasonable request.

\section{Competing interests}

The authors declare that they have no competing interests.

\section{Funding}

This work was supported by the National Natural Science Foundation of China (grant numbers: 30872167). The funding source didn't play a role in the study design, data collection, data analysis, data interpretation, or writing of this manuscript.

\section{Authors' contributions}

Hongzhuan Tan designed and directed the implementation of the study. Ying Zhou conducted the literature review, statistical analysis, and drafted the manuscript. Xun Li contributed to statistical analyses and interpreting the results. Shimin Hu conducted the genotyping and genetic data analyses. Shujin Zhou designed the medical part of the study protocol and supervised the field activities (participant inclusion / exclusion and data collection).

\section{Acknowledgments}

We thank the staffs of the Liuyang Municipal Hospital of Maternal and Child Health for help in acquiring blood samples and medical record information.

\section{References}

1. Mustafa R, Ahmed S, Gupta A, Venuto RC. A comprehensive review of hypertension in pregnancy. Journal of pregnancy.2012;105918.

2. Walker JJ. Pre-eclampsia. Lancet (London, England). 2000; 356(9237):1260-5.

3. Bokslag A, van Weissenbruch M, Mol BW, de Groot CJ. Preeclampsia; short and long-term consequences for mother and neonate. Early Hum Dev. 2016; 102:47-50. 
4. Cavalli Rde C, Sandrim VC, dos Santos JE, Duarte G. [Preeclampsia predition]. Rev Bras Ginecol Obstet. 2009; 31(1):1-4.

5. Salonen Ros H, Lichtenstein P, Lipworth L, Cnattingius S. Genetic effects on the liability of developing preeclampsia and gestational hypertension. Am J Med Genet. 2000; 91(4):256-260.

6. Nilsson E, Salonen Ros H, Cnattingius S, Lichtenstein P. The importance of genetic and environmental effects for pre-eclampsia and gestational hypertension: a family study. Bjog. 2004; 111(3):200-6.

7. Luscher TF. The endothelium. Target and promoter of hypertension? Hypertension. 1990; 15(5):482-5.

8. Intengan HD, Schiffrin EL. Vascular remodeling in hypertension: roles of apoptosis, inflammation, and fibrosis. Hypertension. 2001; 38(3 Pt 2):581-7.

9. Walker JJ. Inflammation and preeclampsia. Pregnancy Hypertens. 2011; 1(1):43-7.

10. Vik KL, Romundstad P, Nilsen TI. Tracking of cardiovascular risk factors across generations: family linkage within the population-based HUNT study, Norway. J Epidemiol Community Health. 2013; 67(7):564-570.

11. Li X, Tan H, Zhou S, Hu S, Zhang T, Li Y, Dou Q, Lai Z, Chen F. Renin-angiotensin-aldosterone system gene polymorphisms in gestational hypertension and preeclampsia: A case-control gene-association study. Sci Rep. 2016; 6:38030.

12. Lu X, Wang L, Lin X, Huang J, Charles Gu C, He M, Shen H, He J, Zhu J, Li H et al. Genome-wide association study in Chinese identifies novel loci for blood pressure and hypertension. Human molecular genetics. 2015; 24(3):865-874.

13. Wang L, Li H, Yang B, Guo L, Han X, Li L, Li M, Huang J, Gu D. The Hypertension Risk Variant Rs820430 Functions as an Enhancer of SLC4A7. American journal of hypertension. 2017;30(2):202-8.

14. Newton-Cheh C, Johnson T, Gateva V, Tobin MD, Bochud M, Coin L, Najjar SS, Zhao JH, Heath SC, Eyheramendy $S$ et al. Genome-wide association study identifies eight loci associated with blood pressure. Nature genetics. 2009; 41(6):666-676.

15. Tranquilli AL, Dekker G, Magee L, Roberts J, Sibai BM, Steyn W, Zeeman GG, Brown MA. The classification, diagnosis and management of the hypertensive disorders of pregnancy: A revised statement from the ISSHP. Pregnancy Hypertens. 2014;4(2):97-104.

16. Brown MA, Lindheimer MD, de Swiet M, Van Assche A, Moutquin JM. The classification and diagnosis of the hypertensive disorders of pregnancy: statement from the International Society for the Study of Hypertension in Pregnancy (ISSHP). Hypertens Pregnancy. 2001; 20(1):9-14.

17. Roberts JM, Hubel CA. The two stage model of preeclampsia: variations on the theme. Placenta. $2009 ; 30$ Suppl A:S32-7.

18. Agrawal S, Singh A. Obesity or Underweight-What is Worse in Pregnancy? J Obstet Gynaecol India. 2016; 66(6):448-452.

19. Sykes SD, Pringle KG, Zhou A, Dekker GA, Roberts CT, Lumbers ER. Fetal sex and the circulating reninangiotensin system during early gestation in women who later develop preeclampsia or gestational hypertension. Journal of human hypertension. 2014; 28(2):133-9.

20. Seely EW, Ecker J. Chronic Hypertension in Pregnancy. New England Journal of Medicine. 2011; 365(5):439-446.

21. Guedes-Martins L. Chronic Hypertension and Pregnancy. Adv Exp Med Biol. 2017; 956:395-407.

22. Daniels D, Ford M, Schwinn M, Benink H, Galbraith M, Amunugama R, Jones R, Allen D, Okazaki N, Yamakawa H. Mutual exclusivity of MED12/MED12L, MED13/13L, and CDK8/19 paralogs revealed within the CDK-Mediator kinase module. J Proteomics Bioinform. 2013; 2(2).

Page $8 / 14$ 
23. Sardar S, Kanne K, Andersson A. Analysis of Polymorphisms in the Mediator Complex Subunit 13-like (Med13L) Gene in the Context of Immune Function and Development of Experimental Arthritis. Archivum immunologiae et therapiae experimentalis. 2018; 66(5):365-377.

24. Blaschke F, Leppanen O, Takata Y, Caglayan E, Liu J, Fishbein MC, Kappert K, Nakayama KI, Collins AR, Fleck E et al. Liver $\mathrm{X}$ receptor agonists suppress vascular smooth muscle cell proliferation and inhibit neointima formation in balloon-injured rat carotid arteries. Circulation research. 2004; 95(12):e110-123.

25. Tomppo L, Ekelund J, Lichtermann D, Veijola J, Järvelin M-R, Hennah W. DISC1 conditioned GWAS for psychosis proneness in a large Finnish birth cohort. PLoS One. 2012; 7(2):e30643.

26. Iwasaki Y, Suganami T, Hachiya R, Shirakawa I, Kim-Saijo M, Tanaka M, Hamaguchi M, Takai-Igarashi T, Nakai $M$, Miyamoto $Y$ et al. Activating transcription factor 4 links metabolic stress to interleukin- 6 expression in macrophages. Diabetes. 2014; 63(1):152-161.

27. Kitajima S, Takahashi C. Intersection of retinoblastoma tumor suppressor function, stem cells, metabolism, and inflammation. Cancer science. 2017; 108(9):1726-1731.

28. Adela R, Borkar RM, Mishra N, Bhandi MM, Vishwakarma G, Varma BA, Ragampeta S, Banerjee SK. Lower Serum Vitamin D Metabolite Levels in Relation to Circulating Cytokines/Chemokines and Metabolic Hormones in Pregnant Women with Hypertensive Disorders. Frontiers in immunology. 2017; 8:273.

29. Krasnyi AM, Gracheva MI, Sadekova AA, Vtorushina VV, Balashov IS, Kan NE, Borovikov PI, Krechetova LV, Tyutyunnik VL. Complex Analysis of Total and Fetal DNA and Cytokines in Blood Plasma of Pregnant Women with Preeclampsia. Bulletin of experimental biology and medicine. 2018; 164(6):721-5.

30. Pushkin A, Kurtz I. SLC4 base (HCO3 -, CO3 2-) transporters: classification, function, structure, genetic diseases, and knockout models. American journal of physiology Renal physiology .2006; 290(3):F580-599.

31. Boedtkjer E, Praetorius J, Matchkov VV, Stankevicius E, Mogensen S, Füchtbauer AC, Simonsen U, Füchtbauer EM, Aalkjaer C. Disruption of Na+, HCO3- cotransporter NBCn1 (slc4a7) inhibits NO-mediated vasorelaxation, smooth muscle Ca2+ sensitivity, and hypertension development in mice. Circulation. 2011; 110.015974.

32. Boedtkjer E, Damkier HH, Aalkjaer C. NHE1 knockout reduces blood pressure and arterial media/lumen ratio with no effect on resting pHi in the vascular wall. The Journal of physiology. 2012; 590(8):1895-1906.

33. Thomsen AB, Kim S, Aalbaek F, Aalkjaer C, Boedtkjer E. Intracellular acidification alters myogenic responsiveness and vasomotion of mouse middle cerebral arteries. Journal of cerebral blood flow and metabolism : Official Journal of the International Society of Cerebral Blood Flow and Metabolism. 2014; 34(1):161-8.

34. Ng FL, Boedtkjer E, Witkowska K, Ren M, Zhang R, Tucker A, Aalkjaer C, Caulfield MJ, Ye S. Increased NBCn1 expression, $\mathrm{Na}+\mathrm{HCO}-\mathrm{co}$-transport and intracellular $\mathrm{pH}$ in human vascular smooth muscle cells with a risk allele for hypertension. Human molecular genetics. 2017; 26(5):989-1002.

35. Ehret GB, Munroe PB, Rice KM, Bochud M, Johnson AD, Chasman DI, Smith AV, Tobin MD, Verwoert GC, Hwang SJ et al. Genetic variants in novel pathways influence blood pressure and cardiovascular disease risk. Nature. 2011; 478(7367):103-9.

36. Boedtkjer E, Bentzon JF, Dam VS, Aalkjaer C. Na+, HCO3-cotransporter NBCn1 increases pHi gradients, filopodia, and migration of smooth muscle cells and promotes arterial remodelling. Cardiovascular research. 2016; 111(3):227-239.

37. Sibai B, Dekker G, Kupferminc M. Pre-eclampsia. Lancet (London, England). 2005; 365(9461):785-799.

38. Sibai BM. Diagnosis and management of gestational hypertension and preeclampsia. Obstetrics and gynecology. 2003; 102(1):181-192. 
39. Simonetti L, Bruque CD, Fernandez CS, Benavides-Mori B, Delea M, Kolomenski JE, Espeche LD, Buzzalino ND, Nadra AD, Dain L. CYP21A2 mutation update: Comprehensive analysis of databases and published genetic variants. Human mutation. 2018; 39(1):5-22.

40. Xu Z, Chen W, Merke DP, McDonnell NB. Comprehensive mutation analysis of the CYP21A2 gene: an efficient multistep approach to the molecular diagnosis of congenital adrenal hyperplasia. The Journal of molecular diagnostics. 2013; 15(6):745-753.

41. Chatuphonprasert W, Jarukamjorn K, Ellinger I. Physiology and Pathophysiology of Steroid Biosynthesis, Transport and Metabolism in the Human Placenta. Frontiers in pharmacology. 2018; 9:1027.

42. Kim YJ, Park HS, Park MH, Suh SH, Pang MG.Oxidative stress-related gene polymorphism and the risk of preeclampsia. European journal of obstetrics, gynecology, and reproductive biology. 2005;119(1):42-6.

43. Coto E, Tavira B, Marin R, Ortega F, Lopez-Larrea C, Ruiz-Ortega M, Ortiz A, Diaz M, Corao Al, Alonso B et al. Functional polymorphisms in the CYP3A4, CYP3A5, and CYP21A2 genes in the risk for hypertension in pregnancy. Biochem Biophys Res Commun. 2010; 397(3):576-9.

44. Luo ZC, An N, Xu HR, Larante A, Audibert F, Fraser WD. The effects and mechanisms of primiparity on the risk of pre-eclampsia: a systematic review. Paediatric and perinatal epidemiology. 2007; 21 Suppl 1:36-45.

\section{Tables}

Table 1. Demographic and clinical characteristics of the study groups.

\begin{tabular}{|c|c|c|c|c|c|c|}
\hline \multirow[t]{2}{*}{ aracteristics } & Control & $\mathrm{GH}$ & \multirow[t]{2}{*}{$P^{\mathrm{a}}$} & $\mathrm{PE}$ & \multirow[t]{2}{*}{$P^{\mathrm{b}}$} & \multirow[t]{2}{*}{$P^{\mathrm{C}}$} \\
\hline & $\mathrm{N}=192$ & $\mathrm{~N}=84$ & & $\mathrm{~N}=108$ & & \\
\hline . ale (n/N (\%)) & $87 / 188(46.28)$ & $46 / 75(61.33)$ & 0.027 & $59 / 103(57.28)$ & 0.073 & 0.587 \\
\hline IN (\%)) & $161 / 192(83.85)$ & $65 / 84(77.38)$ & 0.199 & 79/108(73.15) & 0.026 & 0.502 \\
\hline ge at delivery, week, & $39(38,40)$ & $39(38,40)$ & 0.581 & $38(37,39)$ & $<0.001$ & 0.005 \\
\hline $\mathrm{BP}, \mathrm{mmHg}(\mathrm{M}(\mathrm{QL}, \mathrm{QU}))$ & $110(100,120)$ & $138(120,143)$ & $<0.001$ & $140(130,148)$ & $<0.001$ & 0.003 \\
\hline $\mathrm{BP}$ & $70(60,75)$ & $85(78,92)$ & $<0.001$ & $89(80,95)$ & $<0.001$ & 0.084 \\
\hline \multicolumn{7}{|l|}{ QU) $)^{\mathrm{d}}$} \\
\hline $\mathrm{lg} / \mathrm{dL}(\mathrm{M}(\mathrm{QL}, \mathrm{QU}))$ & $61.65(50.63,69.06)$ & $61.77(58.04,69.99)$ & 0.129 & $67.41(60.37,73.95)$ & $<0.001$ & 0.013 \\
\hline cid, umol/L (mean \pm & $304.89 \pm 99.40$ & $360.06 \pm 97.51$ & $<0.001$ & $395.03 \pm 105.86$ & $<0.001$ & 0.042 \\
\hline :ight, kg (mean \pm SD) & $3.27 \pm 0.41$ & $3.31 \pm 0.50$ & 0.494 & $3.12 \pm 0.68$ & 0.048 & 0.036 \\
\hline
\end{tabular}


Abbreviations: GH, gestational hypertension; PE, preeclampsia; M (QL, QU), Median (lower quartile (25\%), upper quartile (75\%)); SBP, systolic blood pressure; DBP, diastolic blood pressure.

a Comparison of GH and control group.

b Comparison of PE and control group.

c Comparison of GH and PE group.

d In-hospital SBP and In-hospital DBP: BP were measured when pregnant women came to hospital for delivery and before entering the delivery room. GH and PE women might have received treatment to control their BP before measurements.

Table 2. Distributions of tested alleles and genotypes among cases and control subjects.

\begin{tabular}{|c|c|c|c|c|c|c|c|c|c|c|}
\hline \multirow[t]{2}{*}{ Gene } & \multirow[t]{2}{*}{ SNP } & \multirow{2}{*}{$\begin{array}{l}\text { Genotype/ } \\
\text { Allele }\end{array}$} & \multicolumn{2}{|c|}{ Controls } & \multicolumn{2}{|c|}{$\mathrm{GH}$} & \multirow[t]{2}{*}{$P$} & \multicolumn{2}{|c|}{$\mathrm{PE}$} & \multirow[t]{2}{*}{$P$} \\
\hline & & & $\mathrm{n}$ & $\%$ & $\mathrm{n}$ & $\%$ & & $\mathrm{n}$ & $\%$ & \\
\hline \multirow[t]{5}{*}{ MED13L } & rs11067763 & $\mathrm{AA}$ & 72 & 38.9 & 29 & 35.8 & 0.007 & 44 & 41.9 & 0.091 \\
\hline & & AG & 71 & 38.4 & 45 & 55.6 & & 48 & 45.7 & \\
\hline & & GG & 42 & 22.7 & 7 & 8.6 & & 13 & 12.4 & \\
\hline & & A & 215 & 58.1 & 103 & 63.6 & 0.250 & 136 & 64.8 & 0.814 \\
\hline & & $\mathrm{G}$ & 155 & 41.9 & 59 & 36.4 & & 74 & 35.2 & \\
\hline \multirow[t]{5}{*}{$S L C 4 A 7$} & rs820430 & GG & 82 & 44.3 & 32 & 38.6 & 0.052 & 48 & 45.3 & 0.053 \\
\hline & & GA & 94 & 50.8 & 40 & 48.2 & & 45 & 42.5 & \\
\hline & & $\mathrm{AA}$ & 9 & 4.9 & 11 & 13.3 & & 13 & 12.3 & \\
\hline & & $\mathrm{G}$ & 258 & 71.5 & 104 & 62.7 & 0.043 & 141 & 66.5 & 0.436 \\
\hline & & A & 103 & 28.5 & 62 & 37.3 & & 71 & 33.5 & \\
\hline \multirow[t]{5}{*}{$\triangle Y P 21 A 2$} & rs2021783 & $\mathrm{CC}$ & 108 & 56.2 & 50 & 59.5 & $0.884^{*}$ & 54 & 50.0 & 0.200 \\
\hline & & $\mathrm{CT}$ & 66 & 34.4 & 26 & 31.0 & & 38 & 35.2 & \\
\hline & & $\mathrm{TT}$ & 16 & 8.3 & 8 & 9.5 & & 16 & 14.8 & \\
\hline & & $\mathrm{C}$ & 282 & 74.2 & 126 & 75.0 & 0.845 & 146 & 67.6 & 0.113 \\
\hline & & $\mathrm{T}$ & 98 & 25.8 & 42 & 25.0 & & 70 & 32.4 & \\
\hline
\end{tabular}

*P value was calculated by Fisher's exact test. 
Table 3. Logistic regression for SNPs.

Page $12 / 14$ 


\begin{tabular}{|c|c|c|c|c|c|c|c|c|c|}
\hline \multirow[t]{3}{*}{ NP } & \multirow{3}{*}{$\begin{array}{l}\text { Genotype/ } \\
\text { Allele }\end{array}$} & \multicolumn{4}{|c|}{ Univariate logistic regression model } & \multicolumn{4}{|c|}{ Multiple logistic regression model } \\
\hline & & \multicolumn{2}{|r|}{$\mathrm{GH}$} & \multicolumn{2}{|r|}{$\mathrm{PE}$} & \multicolumn{2}{|r|}{$\mathrm{GH}$} & \multicolumn{2}{|r|}{$\mathrm{PE}$} \\
\hline & & OR & $95 \% \mathrm{CI}$ & OR & $95 \% \mathrm{CI}$ & OR* & $95 \% \mathrm{CI}^{*}$ & OR* & $95 \% \mathrm{CI}^{*}$ \\
\hline $13 \mathrm{~L}$ & G vs. A & 0.795 & $(0.543,1.163)$ & 0.755 & $(0.532,1.072)$ & 0.830 & $(0.523,1.474)$ & 0.718 & $(0.499,1.033)$ \\
\hline \multirow[t]{2}{*}{167763} & AG vs. AA & 1.574 & $\begin{array}{l}(0.890) \\
2.783)\end{array}$ & 1.106 & $\begin{array}{l}(0.655) \\
1.869)\end{array}$ & 1.598 & $(0.864,2.959)$ & 0.982 & $(0.570,1.691)$ \\
\hline & GG vs. AA & 0.414 & $\begin{array}{l}(0.167) \\
1.027)\end{array}$ & 0.506 & $\begin{array}{l}(0.245) \\
1.047)\end{array}$ & 0.472 & $(0.184,1.212)$ & 0.497 & $(0.237,1.045)$ \\
\hline $13 \mathrm{~L}$ & $\mathrm{AG}+\mathrm{GG}$ & 1.143 & $(0.665,1.964)$ & 0.883 & $(0.543,1.438)$ & 1.165 & $(0.655,2.074)$ & 0.798 & $(0.483,1.320)$ \\
\hline 167763 & vs. AA & & & & & & & & \\
\hline rant & & & & & & & & & \\
\hline $13 \mathrm{~L}$ & GG vs. & 0.322 & $(0.138,0.752)$ & 0.481 & $(0.245,0.945)$ & 0.376 & $(0.158,0.892)$ & 0.489 & $(0.245,0.979)$ \\
\hline 167763 & $\mathrm{AA}+\mathrm{AG}$ & & & & & & & & \\
\hline sive & & & & & & & & & \\
\hline A7 & A vs. G & 1.373 & $(0.935,2.018)$ & 1.160 & $(0.808,1.665)$ & 1.560 & $(1.037,2.347)$ & 1.278 & $(0.879,1.857)$ \\
\hline 1430 & AG vs. GG & 1.090 & $(0.628,1.892)$ & 0.818 & $(0.494$ & 1.305 & $(0.724,2.354)$ & 0.881 & $(0.523,1.484)$ \\
\hline & & & & & 1.353) & & & & \\
\hline & AA vs. GG & 3.132 & $(1.186,8.271)$ & 2.468 & $(0.982,6.201)$ & 4.367 & $(1.494,12.763)$ & 2.931 & $(1.134,7.574)$ \\
\hline A7 & $\mathrm{AG}+\mathrm{AA}$ & 1.269 & $(0.748,2.153)$ & 0.962 & $(0.595$ & 1.531 & $(0.867,2.704)$ & 1.065 & $(0.647$ \\
\hline 1430 & vs. GG & & & & 1.555) & & & & $1.752)$ \\
\hline rant & & & & & & & & & \\
\hline A7 & AA vs. & 2.988 & $(1.188,7.517)$ & 2.734 & $(1.127$ & 3.562 & $(1.343,9.447)$ & 3.351 & $(1.352$, \\
\hline 1430 & $\mathrm{GG}+\mathrm{AG}$ & & & & 6.632) & & & & 8.303) \\
\hline sive & & & & & & & & & \\
\hline $1 \mathrm{~A} 2$ & T vs. $\mathrm{C}$ & 0.959 & $(0.631,1.457)$ & 1.380 & $(0.957,1.990)$ & 0.874 & $(0.561,1.363)$ & 1.304 & $(0.891,1.908)$ \\
\hline :1783 & CT vs. CC & 0.851 & $(0.484$ & 1.152 & $(0.688$ & 0.745 & $(0.409,1.359)$ & 1.103 & $(0.647$ \\
\hline & & & 1.496) & & 1.929) & & & & $1.881)$ \\
\hline & TT vs. CC & 1.080 & $(0.434$ & 2.000 & $(0.930$ & 0.973 & $(0.370,2.557)$ & 1.389 & $(0.919$ \\
\hline & & & $2.690)$ & & 4.303) & & & & $2.100)$ \\
\hline $1 \mathrm{~A} 2$ & CT+TT vs. & 0.896 & $(0.532$ & 1.317 & $(0.820$ & 0.789 & $(0.454,1.371)$ & 1.239 & $(0.758$ \\
\hline :1783 & $\mathrm{CC}$ & & 1.509) & & $2.116)$ & & & & 2.023) \\
\hline lant & & & & & & & & & \\
\hline $1 \mathrm{~A} 2$ & TT vs. & 1.145 & $(0.470$ & 1.891 & $(0.905$ & 1.087 & $(0.425,2.781)$ & 1.330 & $(0.902$, \\
\hline
\end{tabular}


sive

Abbreviations: $\mathrm{GH}$, gestational hypertension; $\mathrm{PE}$, preeclampsia; $\mathrm{SNP}=$ single nucleotide polymorphism; $\mathrm{OR}=$ odd ratio; $\mathrm{CI}=$ confidence interval.

* Statistics estimated by multiple logistic regression analysis after adjusting for fetal sex and primipara. 